\title{
Fast Handoff Implementation by using Geometrical Mathematical Models and Carrier to Interference Ratio based Handoff Algorithm
}

\author{
Debabrata Sarddar \\ Department of Electronics and \\ Telecommunication Engg, \\ Jadavpur University, Kolkata - \\ 700032
}

\author{
Arnab Raha \\ Department of Electronics and \\ Telecommunication Engg, \\ Jadavpur University, Kolkata - \\ 700032
}

\author{
Shubhajeet Chatterjee \\ Department of Electronics and \\ Communication Engg, Institute of \\ Engg. \& Managment college, \\ saltlake, Kolkata-700091.
}

\author{
Sreya Mallik \\ Department of Electronics and \\ Telecommunication Engg, \\ Jadavpur University, Kolkata - \\ 700032 \\ Mrinal Kanti Naskar \\ Department of Electronics and \\ Telecommunication Engg, \\ Jadavpur University, Kolkata - \\ 700032
}

\author{
Pulak Mazumder \\ Department of Electronics and \\ Telecommunication Engg, \\ Regent Education \& Research \\ Foundation, Barracpore
}

\author{
Utpal Biswas \\ Department of Computer \\ Science and Engg, University of \\ Kalyani, Nadia, West Bengal, \\ Pin- 741235.
}

\begin{abstract}
IEEE $802.11 \mathrm{~b}$ networks are becoming more ubiquitous. While roaming through access points, a Mobile Station is often required to perform a link layer handover. This mechanism causes connection losses and breaks in time-sensitive communication, especially if a network layer handover follows the link layer handover. In this paper, we propose to reduce handoff latency for IEEE 802.11 wireless networks with Neighbor Graphs (NG) pre-scanning mechanisms and using a GPS based server which predetermines the next probable AP using three formu lated geometry based mathematical techniques. IEEE 802.11 uses 11 channels of which the channels 1, 6 and 11 do not mutually overlap. As they are non-overlapping, the channels are expected to have a lower carrier-to-interference ratio (CI) compared to the other channels present under the same base station, which increases the channel's availability during handoff. When handoff criterion have been met, we design an algorithm to first determine the Access Point (AP) under whose coverage area the Mobile Station (MS) would enter, then scanning the channels 1,6 and 11, if present under the next Access Point (AP), to reduce the scanning delay. We also introduce pre-authentication mechanism, which will effectively reduce the message processing delay.
\end{abstract}

\section{Keywords}

Handoff, Neighbor Graph, Access point (AP) Selective channel scanning mechanism, Carrier- to-Interference (CI) Ratio, Global Position Systems (GPS)

\section{INTRODUCTION}

IEEE 802.11b based wireless and mobile networks [1], also called Wi-Fi commercially, are experiencing a very fast growth upsurge and are being widely deployed for providing variety of services as it is cheap, and allows anytime, anywhere access to network data. However they suffer from limited coverage ran ge of AP, resulting in frequent handoffs, even in moderate mobility scenarios. Handoff, an inherent problem with wireless networks, particularly real time applications, has not been well addressed in IEEE 802.11, which takes a hard handoff approach [2]. Here a mobile host $(\mathrm{MH})$ has to break its connection with its old access point (AP) before connecting to a new AP, resulting in prolonged handoff latency called link switching delay. Now-adays, soft handoff procedure is in use. Here a Mobile Station is connected to its old AP till it makes connection with the new AP. This effectively reduces the packet losses incurred by hard handoff.

With the advent of real time applications, the latency and packet loss caused by mobility became an important issue in Mobile Networks. The most relevant topic of discussion is to reduce the IEEE 802.11 link-layer handoff latency. IEEE 802.11 MAC specification [3] defines two operation modes: ad hoc and infrastructure mode. In the ad hoc mode, two or more stations (STAs) recognize each other through beacons and hence establish a peer-to-peer relationship. In infrastructure mode, an AP provides network connectivity to its associated STAs to form a Basic Service Set (BSS). Multiple APs form an Extended Service Set (ESS) that constructs the same wireless networks. 
The complete handoff procedure can be divided into 3 distinct logical parts: scanning, authentication and re-association. In the first phase, an STA scans for APs by either sending Probe Request messages or by listening for beacon message. After scanning all channels, an AP is selected using the Received Signal Strength Indication (RSSI) and CI ratio, and the selected AP exchanges IEEE 802.11 authentication messages with the STA. Finally, if the AP authenticates the STA, the STA sends Re-association Request message to the new AP.

The overall delay is the summation of scanning delay, authentication delay, and re-association delay. According to [4], $90 \%$ of handoff delay comes from scanning delay. The range of scanning delay is given by:-

$$
\mathrm{N} \times \mathrm{T}_{\min } \leq \mathrm{T}_{\text {scan }} \leq \mathrm{N} \times \mathrm{T}_{\max }
$$

Where $\mathrm{N}$ is the total number of channels according to the spectrum released by a country, $\mathrm{T}_{\min }$ is Min Channel Time, $\mathrm{T}_{\text {scan }}$ is the total measured scanning delay, and $\mathrm{T}_{\max }$ is Max Channel Time. Here we focus on reducing the scanning delay. We divide our paper into the following sections: Section-2 discusses the related works in this field; the proposed method is explained in Section-3. Section-4 discusses our simulations and experimental results, followed by conclusions and future works, and finally the references.

\section{RELATED WORKS}

Besides, much progress has been made in introducing GPS aided handoffs; vide [10], [11], [12], [13], [14]. In our work also we look at another such position dependant solution with a view to minimize overhead signalling problems. This is necessary since extensive pre-scanning is unacceptable in high traffic AP dense networks.Most of the related works focus on reducing the handover delay in the scan phase, as delay in the potential phase is hardware dependent and in the authentication phase is negligible in an open wireless environment. The process of AP scan and reconnection is intolerably slow, of the order of 200$300 \mathrm{~ms}$ or even longer, of which $80-90 \%$ delay is attributed to probe delay [3]. In real scenario, it is seen that maximum handoff latency for Voice over IP (VoIP) applications is $50 \mathrm{~ms}$ [8]. To reduce handoff latency in wireless LAN using IAPP [9], an algorithm on context transfer mechanism using 'Neighbor Graph' (NG) [10] was suggested in [9] and in [11] neighbor graph cache mechanism was proposed. However, IAPP was only reactive in nature and creates an additional delay in a handoff. One approach on Physical layer (PHY) is the method using two trans-receivers, where a wireless Mobile Station (MS) has two Wireless Network Interface Cards (WNICs) [3], one for keeping connection to current $\mathrm{AP}$ and the other for scanning channels to search for alternate APs [12]. In [13] the idea of selective scanning and caching mechanism was proposed and the idea of background scanning, pre-authentication and server based restricted channel set was given. Beside that the concept of implementing a hy steresis constant in addition to RSSI threshold was introduced, which is a very important tool to avoid the "toggling effect". In [5], S. Kyriazakos et al. proposed an algorithm to resolve the well-known ping-pong and faraway cell effects using the MS's movement and its velocity. J Persola et al in [6] present a location assisted algorithm to manage handover between WLAN and GPRS networks. Authors of [14] aimed at reducing the authentication process which contributes very little to the handoff time. In [15] a channel mask scheme was introduced where a selective scanning algorithm was proposed along with a caching mechanism. In [16] \& [17], authors use GPS based access point maps for handoff management.

\section{PROPOSED WORK}

In this paper, we propose a selective scanning mechanism using NG to solve the problem of handoff latency. CI ratio forms an essential part in channel selection process during handoff, though traffic plays a more dominant role. IEEE $802.11 \mathrm{~b}$ uses 11 channels out of 14 possible channels, of which only channels $1,6,11$ do not mutually overlap. So these channels will have a very low interference with other frequency. It may have a noticeable interference only if the same channel is used by any other APs within its frequency re-use range. Thus it is quite evident that these channels will have a high probability of having a greater CI ratio as compared to the other channels within the same AP. So based on this fact, when the MS responds to handoff, according to the pre-scanning mechanism of NG, it first looks for the potential AP and then first scans the channels 1, 6 and 11, if present. If this fails, it will start scanning the other channels. In addition, we propose to reduce the authentication delay by pre-authentication method, where the authentication process is performed during the scan phase. More over we consider a hexagonal packed cluster formed by coverage area of 7 APs to find out the most potential AP, towards which the MS heads when it tries to handover its call with the aid of GPS server. Reduction of scanning of the channels of the APs reduces the handover delay to a greater extend. We have formulated three mathematical methods based co-ordinate geometry in two dimensions by virtue of which we can find out the most potential AP towards whose coverage area the MS may enter after handover. The methods are explained in detail in the section to follow.

The maximum range up to which the signal can be transmitted is determined by the height of the antenna and the power of the signal is inversely proportional to the square of the distance from the AP. But due to fading, the signal strength is never equally spread in all direction even for an omni-directional antenna. There are mainly two types of fading responsible for the uneven distribution of the signal strength from the AP. They are namely fast fading (fading due to scattering of the signal by object near transmitter) and slow fading (fading due to long term spatial and temporal variations). Ideally without fading, the cell's coverage area would be circular, but due to fading it becomes an undefined contour. Signal strength contours for two APs operating in ideal condition without fading is given in Figure 1 (a) and operating in real condition with fading is given in Figure 1 (b). Here we define the coverage area of each AP to be concentrated within a hexagon of certain edge length, which is the best approximation so far considering uneven distribution of signal. Second generation wireless systems and most of the research works follow Frequency Division Multiple Access (FDMA) or Time Division Multiple Access (TDMA) for multiple access of a single channel frequency band. In TDMA, one channel is used by several users, with AP assigning time slots for different users, and each user is served in a round-robin method. In FDM A, the allocated frequency band for one channel is subdivided into many sub-bands and each sub-band is allocated by the AP to each user. Thus, in FDMA, it may be seen that a particular sub-band is allocated to a user which falls between the interference zones of channels within the same AP. Thus, protocols using FDMA techniques have a certain probability that during handoff, even when the channel is free, 
the user is allocated such a sub-band within the above mentioned region. Thus, it will encounter a very low $\mathrm{CI}$ ratio and the MS ceases to operate on that channel and scans for the next channel. During scanning of the non over-lapping channels, this problem will not be faced. So our method works even better in cases where FDM A is used for multiple access of a single channel.

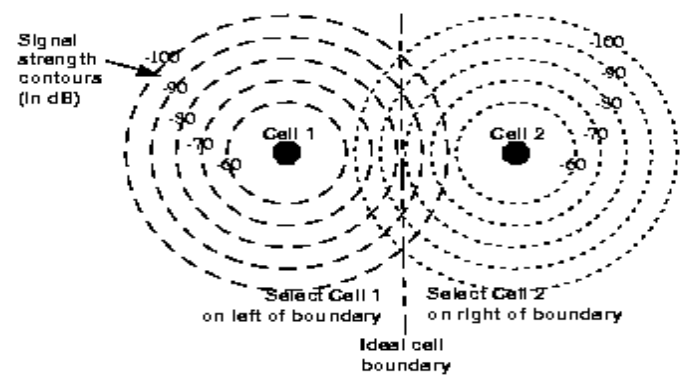

Figure 1 (a). It shows the distribution of signal strength of APs in ideal condition.

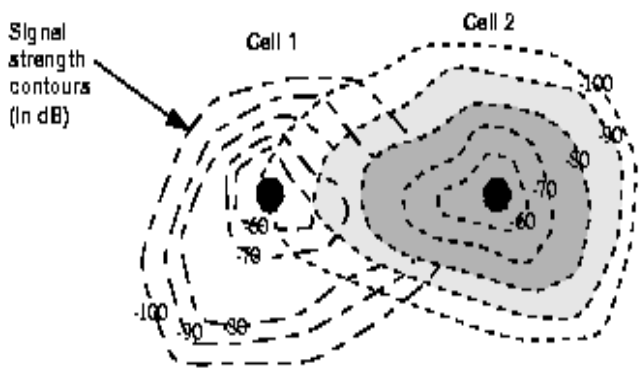

Figure 1 (b). It shows the distribution of signal strength of APs in real condition.

With these assumptions, we propose (i) a selective channel scan, as in [3], and (ii) pre-authentication scheme with the help of neighbor graph for reducing the total handoff delay.

3.1. Selective Channel Scanning: As in [3], the MS downloads from the server the data which not only contains the neighbor of the AP on which it is presently operating, but also the channels used by the neighboring APs. However the MS must wait for min channel time or max channel time as the MS does not know how many APs would respond to the probe request. So here we use unicast instead of broadcast which selects the potential APs to which the call may be handed off and scans only the channels associated with those APs. Selective channel probing with the help of unicast instead of broadcast brilliantly reduces the handoff delay by a massive percentage when compared with selective scanning or basic active scanning. Moreover, it was also stated that the MS has to wait for only the 'round trip time' ( $\mathrm{rtt}$ ) for scanning each channel instead of the min channel time or the max channel time. We know that IEEE uses 11 out of the 14 possible channels, out of which 1, 6 and 11 are mutually non-overlapping. When the MS responds to handoff, according to the pre-scanning mechan ism of NG, it first looks for the potential AP and then first scans the channels 1, 6 and 11 if present. If this fails, it will start scanning the other channels. As proposed in [3], the expected scanning delay using selective scanning is

$$
\mathrm{t}=\mathrm{N}^{\prime} \times \tau+\alpha,
$$

where ' $\mathrm{t}$ ' is the scanning delay, $\mathrm{N}$ ' is the number of channels scanned, ' $\tau$ ' is the round trip time and $\alpha$ is the message processing time. ' $\tau$ ' is the summation of the time taken for the Probe Request to be sent to the selected APs and for the Probe Response to be received, which, in our case, is nothing but the Min Channel Time, which has been estimated to be around 3-7 ms.

\subsection{The Angular Dis placement Method:}

The entire hexagonal cell is divided into 6 sectors with each sector having an angle of $60^{\circ}$ as shown in the Figure 2. The sectors are numbered serially from 1 to 6 and the angles of each sector being an arithmetic progression with first term and common difference being $60^{\circ}$. So it can be visualized as a two dimensional space with the origin as the AP having 4 quadrants about it. Let the initial coordinate of the MS be $\left(x_{a}, y_{a}\right)$, with origin as the AP and the $\mathrm{X}$ and $\mathrm{Y}$ axis as shown in Figure 2. It notes the angular displacement for every $5 \mathrm{~ms}$ interval in the initialization phase.

Here the term angular displacement is defined as follows. If at the start of any period ' $\mathrm{i}$ ' the coordinate is $\left(\mathrm{x}_{\mathrm{i}}, \mathrm{y}_{\mathrm{i}}\right)$ and at the end $\left(\mathrm{x}_{\mathrm{i}+1}, \mathrm{y}_{\mathrm{i}+1}\right)$ then the angular displacement ' $\Theta_{\mathrm{i}}$ ' at that interval of time is given by

$$
\Theta_{\mathrm{i}}=90^{0} * \mathrm{n}+\bmod \left(\left(\mathrm{y}_{\mathrm{i}+1}-\mathrm{y}_{\mathrm{i}}\right) /\left(\mathrm{x}_{\mathrm{i}+1}-\mathrm{x}_{\mathrm{i}}\right)\right)
$$

Where $n=0$, if $x_{i+1}>=x_{i}$ and $y_{i+1}>=y_{i} ; n=1$, if $x_{i+1}<x_{i}$ and $\mathrm{y}_{\mathrm{i}+1}>\mathrm{y}_{\mathrm{i}} ; \mathrm{n}=2$, if $\mathrm{x}_{\mathrm{i}+1}<\mathrm{x}_{\mathrm{i}}$ and $\mathrm{y}_{\mathrm{i}+1}<\mathrm{y}_{\mathrm{i}}, \mathrm{n}=3$, if $\mathrm{x}_{\mathrm{i}+1}>\mathrm{x}_{\mathrm{i}}$ and $\mathrm{y}_{\mathrm{i}+1}<\mathrm{y}_{\mathrm{i}}$. The net angular displacement ' $\Psi$ ' after any time interval ' $i$ ' is given by the equation:

$$
\Psi=90^{0} * \mathrm{n}+\bmod \left(\left(\mathrm{y}_{\mathrm{i}}-\mathrm{y}_{\mathrm{a}}\right) /\left(\mathrm{x}_{\mathrm{i}}-\mathrm{x}_{\mathrm{a}}\right)\right)
$$

Where $\mathrm{n}=0$, if $\mathrm{x}_{\mathrm{i}}>=\mathrm{x}_{\mathrm{a}}$ and $\mathrm{y}_{\mathrm{i}}>=\mathrm{y}_{\mathrm{a}} ; \mathrm{n}=1$, if $\mathrm{x}_{\mathrm{i}}\left\langle\mathrm{x}_{\mathrm{a}}\right.$ and $\mathrm{y}_{\mathrm{i}}>\mathrm{y}_{\mathrm{a}}$; $\mathrm{n}=2$, if $\mathrm{x}_{\mathrm{i}}<\mathrm{x}_{\mathrm{a}}$ and $\mathrm{y}_{\mathrm{i}}<\mathrm{y}_{\mathrm{a}} ; \mathrm{n}=3$, if $\mathrm{x}_{\mathrm{i}}>\mathrm{x}_{\mathrm{a}}$ and $\mathrm{y}_{\mathrm{i}}<\mathrm{y}_{\mathrm{a}}$.

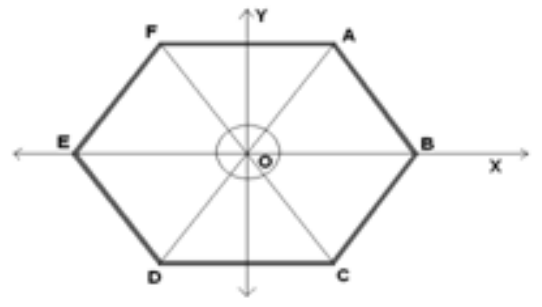

Figure 2. The figure showing the $\mathrm{X}$ and $\mathrm{Y}$ axes description for the model

Even after initialization the angular displacement and the net angular displacement are carried on at an interval $5 \mathrm{~ms}$, thus reserving time for calculation of the various parameters. Another parameter, which is the average rate of change of angular displacement after any time interval is given by the ratio of the angular displacement at each time period to the total time of observation. So this parameter designated as $\Theta^{\prime}$ is given by the equation:

$$
\Theta^{\prime}=\left(\sum \Theta_{\mathrm{i}}\right) /(5 * \mathrm{i})
$$

When the MS falls in the shaded region as denoted in Figure 2, the given mechanism stops and we can determine the AP to which it will head to by finding the angle ' $\Lambda$ ' which is given by:

$$
\Lambda=\Psi+\Theta^{\prime} * t_{\text {delay }}
$$

Where $\Psi$ and $\Theta^{\prime}$ is the value recorded at the last interval of measurement. Now for the various values of ' $\Lambda$ ', the MS would head towards a certain AP as shown in Figure 3. So the potential AP can be found and the channels corresponding to that AP can be scanned, which thus efficiently reduces the handoff latency. To make the approximation more accurate, we calculate the error in the co-ordinate estimation after each time interval. This 
is carried on after the initialization phase where the expected angle at the end of the $i^{\text {th }}$ interval is compared to the actual angle after the $\mathrm{i}^{\text {th }}$ interval. For the net an gular displacement, there are two errors i.e. the maximum positive error and the maximum negative error. They are denoted by the parameter $e_{p}$ and $e_{n}$ respectively for positive and negative values. These are recorded by any sorting algorithm or simply by comparing the error after any time interval with the present values. These values come into action at the moment of determination of the potential AP. Now the expected range of net angular displacement, if ' $i$ ' no of time intervals has been observed, is given as:

$$
\Lambda-e_{n}<=\text { Net angular displacement }<=\Lambda+e_{p}
$$

So we get a range of value for the net angular displacement. It is shown in the simulation part that it yields at the most two APs within this range but gives a higher accuracy corresponding to handover. But most of the time, the algorithm gives one potential AP, and in maximum of those cases, the handoff is favorable by scanning the channels corresponding to the most potential AP only. The simulation of algorithm is provided in the next section with a detailed performance evaluation which judges the suitability of this algorithm in real conditions.
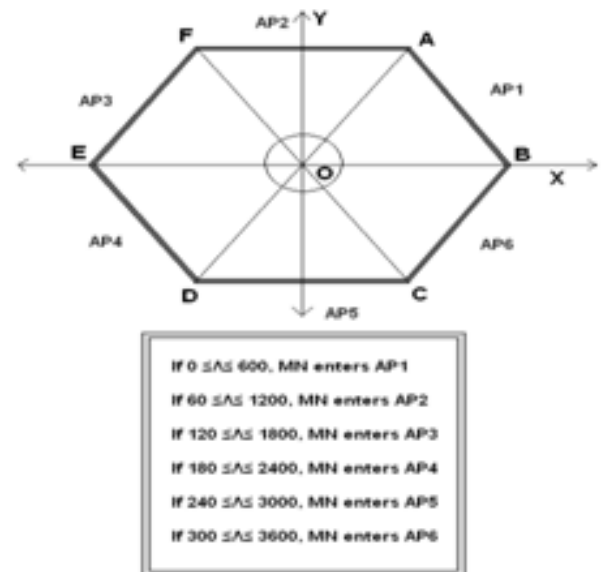

Figure 3. The figure and chart giving details of the various angles which gives the direction and the AP towards which the MS is expected to get its call handed over

3.3. Co-ordinate Evaluation Method: The information regarding the latitude and longitude of the MS can be easily got by GPS. After any time interval 'i' let the latitude be lat ${ }_{i}$ and the longitude long. Now this latitude and longitude evaluation is done at each interval from the beginning of the initialization phase and after that with a period of $5 \mathrm{~ms}$. The coordinate displacement is defined as the change in latitude and longitude after a given time period. It is calculated after any time interval ' $i$ ' as the difference in latitudinal co-ordinate equal to "lat $\mathrm{i}_{\mathrm{i}}-\mathrm{lat}_{\mathrm{i}-1}$ " and the lon gitudinal co-ordinate " $\operatorname{long}_{\mathrm{i}}-\operatorname{long}_{\mathrm{i}-1}$ ". This are written as $\Delta$ lat $_{\mathrm{i}}$ and $\Delta$ long $_{\mathrm{i}}$ respectively after any time interval ' $t$ '. The co-ordinate displacement after each time period is noted down. A new parameter named as the average rate of change of co-ordinate is framed which gives the average of all the co-ordinate displacement corresponding to both the latitude and longitude divided by the total time of observation. These are denoted by the symbols $\lambda_{\text {lat }}$ and $\lambda_{\text {long }}$ for latitude and longitude respectively and after any time interval ' $i$ ' they are given as,

$$
\lambda_{\text {lat }}=\left(\sum \Delta \mathrm{lat}_{\mathrm{i}}\right) /(5 * \mathrm{i})
$$

$\lambda_{\text {long }}=\left(\sum \Delta \operatorname{long}_{\mathrm{i}}\right) /(5 * \mathrm{i})$

Where summation is carried for all integer values starting from 1 to i. So whenever the MS enters the shaded region as shown in Figure 8 , the given algorithm stops. If ' $i$ ' number of time intervals have been observed then the co-ordinate of the MS after the time $t_{\text {delay }}$ is expected to be given as $\left(\left(\right.\right.$ lat $\left._{\mathrm{i}}+\lambda_{\text {lat }} * \mathrm{t}_{\text {delay }}\right)$, $\left(\right.$ long $\left.\left._{\mathrm{i}}+\lambda_{\text {long }} * t_{\text {delay }}\right)\right)$. By the knowledge of the expected coordinate of the MS after the handoff process, it can be expected towards which AP the MS would be heading. So the most potential AP may be scanned, hence the net handoff latency is reduced by a great margin. To make the approximation more accurate, we calculate the error in the co-ordinate estimation after each time interval. This is carried on after the initialization phase where the expected co-ordinate at the end of the $i^{\text {th }}$ interval is compared to the actual co-ordinate after the $i^{\text {th }}$ interval. For each latitude and longitude, two errors i.e., the maximum positive error and the maximum negative error are calculated. These are recorded by any sorting algorithm or simply by comparing the error after any time interval with the present values. Let the positive error corresponding to latitude be $\mathrm{pe}_{\mathrm{lat}}$ and for longitude be $\mathrm{pe}_{\text {long }}$ and correspondingly for negative error let these be $\mathrm{ne}_{\text {lat }}$ and $\mathrm{ne}_{\text {long. These values come }}$ into action at the moment of determination of the potential AP. Now the expected range of co-ordinate if ' $i$ ' no of time intervals have been observed is given as:

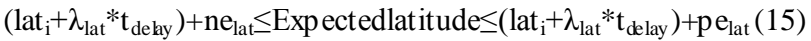
(long $\left.{ }_{\mathrm{i}}+\lambda_{\text {long }} * \mathrm{t}_{\text {delay }}\right)+$ ne $_{\text {long }}<=$ Expectedlongitude $<=\left(\right.$ long $_{\mathrm{i}}+\lambda_{\text {long }} * \mathrm{t}_{\text {dela }}$ y) + pe long $\quad \ldots . .(16)$

So we get a range of values for latitude and longitude. It is shown in the simulation part that it yields at the most two APs within this range but gives a higher accuracy corresponding to handover. But most of the time, the algorithm gives one potential AP, and in maximum of those cases, the handoff is favorable by scanning the channel corresponding to the most potential AP only. The simulation of algorithm is provided in the next section with a detailed performance evaluation which judges the suitability of this algorithm in real conditions.

3.4. Area Comparison Method: Let us consider an isotropic point source fed by a transmitter of power $\mathrm{P}_{\mathrm{t}}$ watts. At an arbitrary large distance $d$ from the source, the radiated power is uniformly distributed over the surface area. The received signal power at a distance $d$ is given by $P_{r}=\left(A_{e} \times G_{t} x\right.$ $\left.P_{t}\right) /\left(4 \times\right.$ pi x d $\left.{ }^{2}\right)$. Where $A_{e}=$ effective area and $G_{t}=$ transmitting antenna gain. The relationship between an effective aperture and the receiving antenna gain $G_{r}$ is given as $G_{r}=\left(4 \times p i x A_{e}\right) / y^{2}$. Where $y$ is the wavelength of the electromagnetic wave. Combining the two relations we get:

$\mathrm{P}_{\mathrm{r}}=\left(\mathrm{G}_{\mathrm{r}} \times \mathrm{G}_{\mathrm{t}} \times \mathrm{P}_{\mathrm{t}}\right) /((4 \mathrm{x} \mathrm{pixd}) / \mathrm{y})^{2}$

If we consider uniform topology, then the region of influence of the signal transmitted from an AP can be approximated as a circular region.For any mathematical or practical purpose the coverage area of an AP is considered to be hexagonal with the AP situated at the centre of the hexagon and this hexagonal cell is surrounded by six other similar hexagonal cells (considering the antenna strength of each of these cells to be equal). If we consider that centering one AP there are six other APs, then each of them has got a circle of influence on the basis of the strength of the transmitted signal. The intersection of the seven circles eventually gives six points equidistant from the central AP. These points when joined gives rise to a hexagon. These individual cells form the seven cell cluster as shown in Figure 4. 
Here we will consider the N-S and the E-W directions as the two directional axes perpendicular to each other and compare it with the $x-y$ axes of the rectangular co-ordinate system. From Figure 5 we get the co-ordinates of the six vertices of the hexagon $\mathrm{P}_{1} \mathrm{P}_{2} \mathrm{P}_{3} \mathrm{P}_{4} \mathrm{P}_{5} \mathrm{P}_{6}$ as $\mathrm{P}_{\mathrm{i}}=\left(\mathrm{x}_{\mathrm{i},} \mathrm{y}_{\mathrm{i}}\right)$, for $\mathrm{i}=[1,6]$. With the help of these six co-ordinates the equation of the six edges of the hexagon are found out as follows:

$\left(\mathrm{y}-\mathrm{y}_{\mathrm{i}}\right) /\left(\mathrm{x}-\mathrm{x}_{\mathrm{i}}\right)=\left(\mathrm{y}_{\mathrm{i}}-\mathrm{y}_{\mathrm{i}+1}\right) /\left(\mathrm{x}_{\mathrm{i}-\mathrm{x}} \mathrm{x}_{\mathrm{i}+1}\right)$

The above equation on simplification yields

$\Rightarrow \mathrm{y}=\mathrm{y}_{\mathrm{i}}+\left(\left(\mathrm{y}_{\mathrm{i}}-\mathrm{y}_{\mathrm{i}+1}\right) /\left(\mathrm{x}_{\mathrm{i}}-\mathrm{x}_{\mathrm{i}+1}\right)\right) *\left(\mathrm{x}-\mathrm{x}_{\mathrm{i}}\right)$

$\Rightarrow \mathrm{y}=\left(\left(\mathrm{y}_{\mathrm{i}}-\mathrm{y}_{\mathrm{i}+1}\right) /\left(\mathrm{x}_{\mathrm{i}}-\mathrm{x}_{\mathrm{i}+1}\right)\right) * \mathrm{x}+\left(\mathrm{y}_{\mathrm{i}}-\mathrm{x}_{\mathrm{i}}^{*}\left(\left(\mathrm{yi}_{-} \mathrm{y}_{\mathrm{i}+1}\right) /\left(\mathrm{x}_{\mathrm{i}}-\mathrm{x}_{\mathrm{i}+1}\right)\right)\right)$

$\Rightarrow\left(\left(\mathrm{y}_{\mathrm{i}+1}-\mathrm{y}_{\mathrm{i}}\right) /\left(\mathrm{x}_{\mathrm{i}+1}-\mathrm{x}_{\mathrm{i}}\right)\right)^{*} \mathrm{x}+\mathrm{y}=\left(\left(\mathrm{x}_{\mathrm{i}+1} \mathrm{y}_{\mathrm{i}}-\mathrm{x}_{\mathrm{i}} \mathrm{y}_{\mathrm{i}+1}\right) /\left(\mathrm{x}_{\mathrm{i}+1}-\mathrm{x}_{\mathrm{i}}\right)\right)$

$\Rightarrow\left(\left(\mathrm{y}_{\mathrm{i}+1}-\mathrm{y}_{\mathrm{i}}\right) /\left(\mathrm{x}_{\mathrm{i}+1} \mathrm{y}_{\mathrm{i}}-\mathrm{x}_{\mathrm{i}} \mathrm{y}_{\mathrm{i}+1}\right)\right)^{*} \mathrm{x}+\left(\left(\mathrm{x}_{\mathrm{i}+1}-\mathrm{x}_{\mathrm{i}}\right) /\left(\mathrm{x}_{\mathrm{i}+1} \mathrm{y}_{\mathrm{i}}-\mathrm{x}_{\mathrm{i}} \mathrm{y}_{\mathrm{i}+1}\right)\right)^{*} \mathrm{y}$

$=1$

$\Rightarrow \mathrm{ax}+\mathrm{by}=1$

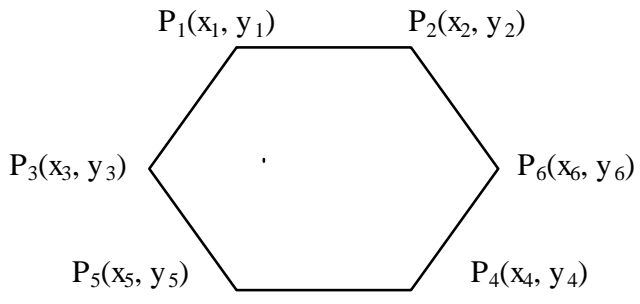

Figure 4.Coordinate of each edge of the approximated hexagonal cell coverage area with the AP denoted as the origin $\mathrm{O}$

Where, $a=\left(\left(y_{i+1}-y_{i}\right) /\left(x_{i+1} y_{i}-x_{i} y_{i+1}\right)\right)$

And, $\mathrm{b}=\left(\left(\mathrm{x}_{\mathrm{i}+1}-\mathrm{x}_{\mathrm{i}}\right) /\left(\mathrm{x}_{\mathrm{i}+1} \mathrm{y}_{\mathrm{i}}-\mathrm{x}_{\mathrm{i}} \mathrm{y}_{\mathrm{i}+1}\right)\right)$

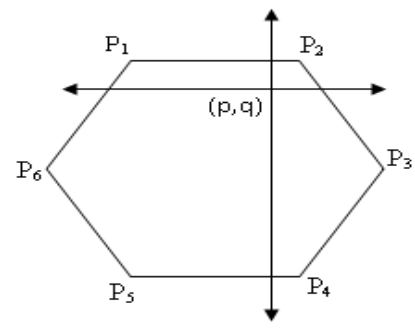

Figure 5. The $x$ axes and $y$ axes drawn relative to the point $(\mathbf{p}, \mathbf{q})$

As soon as the MS enters a hexagon, the current co-ordinate of the MS is obtained with the aid of GPS. Let the current position be given by $(p, q)$. Taking it as the origin we obtain two straight lines:

$\mathrm{x}=\mathrm{p}$ (parallel to the $\mathrm{N}-\mathrm{S}$ direction)

$\mathrm{y}=\mathrm{q}$ (parallel to the $\mathrm{E}-\mathrm{W}$ direction)

This is shown in Figure 13.

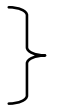

From (18) we can write:

$\mathrm{y}=\mathrm{m}_{1} \mathrm{x}+\mathrm{c}_{1}$

Where $\mathrm{m}_{1}=\left(\left(\mathrm{y}_{\mathrm{i}}-\mathrm{y}_{\mathrm{i}+1}\right) /\left(\mathrm{x}_{\mathrm{i}}-\mathrm{x}_{\mathrm{i}+1}\right)\right)$,

And,

$$
c_{1}=\left(\left(x_{i+1} y_{i}-x_{i} y_{i+1}\right) /\left(x_{i+1}-x_{i}\right)\right)
$$

$\mathrm{x}=\mathrm{m}_{2} \mathrm{y}+\mathrm{c}_{2}$.

Where $\mathrm{m}_{2}=\left(\left(\mathrm{x}_{\mathrm{i}+1}-\mathrm{x}_{\mathrm{i}}\right) /\left(\mathrm{y}_{\mathrm{i}+1}-\mathrm{y}_{\mathrm{i}}\right)\right)$,

$$
c_{2}=\left(\left(x_{i} y_{i+1}-x_{i+1} y_{i}\right) /\left(y_{i+1}-y_{i}\right)\right)
$$

Putting $\mathrm{x}=\mathrm{p}$ and $\mathrm{y}=\mathrm{q}$ in (20) and (21) respectively six set of coordinates will be obtained for each of the two equations (20) and (21). But we need only the four points which lie on the perimeter of the hexagon
For the selection of these four points the following algorithm is followed:

1. The six vertices of the hexagon are taken and two consecutive vertices are taken at a time.

2. The 12 points obtained are taken each at a time.

3. It is compared if the co-ordinates of the point lie within the co-ordinates of the two vertices.

4. For e.g if the co-ordinate of the point be $\left(\mathrm{p}_{\mathrm{i}}, \mathrm{q}_{\mathrm{i}}\right)$, then it is checked if :

$\left.\mathrm{x}_{\mathrm{i}} \leq \mathrm{p}_{\mathrm{i}} \leq \mathrm{x}_{\mathrm{i}+1}\right\}$

$\left.\mathrm{y}_{\mathrm{i}} \leq \mathrm{q}_{\mathrm{i}} \leq \mathrm{y}_{\mathrm{i}+1}\right\}$

5. If it lies then, that point will be stored ;otherwise it will be deleted.

Following the above algorithm the four points will be obtained. Then the area of the four parts intercepted between the two directional axes and the hexagon is found out. The co-ordinates that is required for the computation of each area is determined as follows:

1. Start with any one of the six vertices of the hexagon.

2. Observe if the vertex has been utilised to save any of the four points obtained from the previous algorithm.

3. If the vertex has such points on both sides (with respect to the adjacent vertices), then close the loop by taking the co-ordinates of the vertex, the two points and the current location of the MS.

4. If the vertex has one such point, then the search operation is shifted to the vertex on that side of the current vertex in which none of the four points were found. Then the same algorithm is carried in a recursive manner until another point of the four points is obtained.

5. The area is calculated by taking the co-ordinates of the two points, the intermediate vertices and the current location of the MS.

6. When the points are selected the corresponding areas are calculated using the formula as given by $0.5 *\left[\sum\left(\mathrm{x}_{\mathrm{i}} \mathrm{y}_{\mathrm{i}+1}-\mathrm{x}_{\mathrm{i}+1} \mathrm{y}_{\mathrm{i}}\right.\right.$ )$\left.+\left(\mathrm{x}_{\mathrm{n}} \mathrm{y}_{1}-\mathrm{x}_{1} \mathrm{y}_{\mathrm{n}}\right)\right]$, where $\mathrm{i}=[1, \mathrm{n}-1]$, where $\mathrm{n}$ is the number of points required to specify an area.

7. Four such areas are calculated and compared to find the least among them. Then it becomes obvious that the handoff procedure will occur through that part of the cell-boundary that constitutes a part of the perimeter of the minimum area calculated.

To predict the possible direction of approach of the MS the following algorithm has to be followed:

1. The slopes of the six edges of the hexagon are calculated in advance whenever an MS enters a BSS. $M_{i}=\left(y_{i}-y_{i+1}\right) /\left(x_{i}-\right.$ $\left.x_{i+1}\right)$, for $n=[1,7]$ and $i=n$ mod 6 when $n=1,2,3,4,5,7$, and $=6$ when $\mathrm{n}=6$

2. The slopes of the sides of the four intercepted areas are also found out and then compared with the above equation.

3. The slope of the side of the hexagon, as shown in Figure 6, with which the slope of any side of the area matches determines the predicted target AP of the MS. Thereby the MS will have to scan the channels of only that AP and thereby the handoff latency will be reduced in totality.

However two situations may arise.

Case 1: The smallest area may contain parts of two sides of the hexagonal cells shown in Figure 6. This is the worst case.

For the case shown in Figure 6, from Table 1, we can conclude that the MS has a probability to move into either AP1 or AP2.

Case2: The smallest area may contain parts of only one edge of the hexagonal cell. This is the best case and is shown in Figure 
11. For this case, from Table1, we can conclude that the MS has a probability to move into AP2.

On the basis of Figure 7 and Figure 8 the predicted target APs are tabulated below:

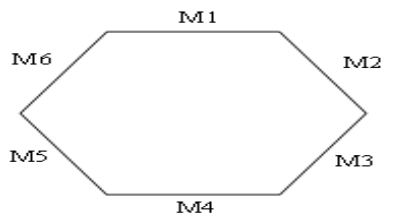

Figure 6. Slopes of the six sides of the hexagon. Table1. Prediction table

\begin{tabular}{|l|l|}
\hline SLOPE & TARGET AP \\
\hline M1 & AP1 \\
\hline M2 & AP2 \\
\hline M3 & AP3 \\
\hline M4 & AP4 \\
\hline M5 & AP5 \\
\hline M6 & AP6 \\
\hline
\end{tabular}

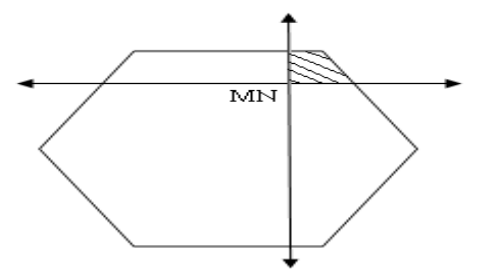

Figure 7. The shaded area represents the direction towards which the MS is expected to switch over taking the consideration of Case 1 .

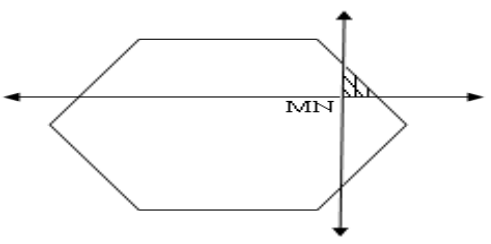

Figure 8. The shaded area represents the direction towards which the MS is expected to switch over taking the consideration of Case 2.

The GPS server chooses the new AP either from two options (as in case 1) or from one option (as in case 2). The server sends a handoff initiate (HI) message to the MS and provides parameters like the target AP's IEEE 802.11 channels, SSID (service set identifier).

This entire handoff process starts after the signal strength received by the MS becomes less than a certain threshold value which depends upon the antenna strength of the AP. But the signal strength of the APs changes rapidly with space and time. It might happen that just after handoff the signal strength of the old AP is better than the current AP and thus the station initiates a handoff with the destination as the old AP. This effect is called the "toggling effect". To avoid this toggling we have added a mechanism of hysteresis i.e. the signal strength of the new AP must be better than the old AP by at least a hysteresis constant. Thus unnecessary handoffs can be reduced with the use of hysteresis. The parameters RSSI threshold and hysteresis constant are configurable and depends on the power of the transmitting signal. As soon as the RSSI received by the MS goes below the threshold, then the minimum area is calculated, consequently the best AP is selected and the scanning phase starts followed by the authentication phase. Just at the moment at which the signal strength of the target AP becomes more than the old AP then by the hysteresis constant the process of reassociation starts - thereby completing the handoff mechanism.

\section{SIMULATION RESULTS}

We first simulate the efficiency of the proposed mathematical models in determining the next AP. For the area comparison method we have made a sample run of our algorithm to test its functional it. The coverage region of the AP is taken as the regular hexagon of side equal to $200 \mathrm{~m}$ (which satisfies the topological conditions of an AP in urban areas). At the end of the algorithm we compare the prediction of our algorithm with the actual result and thereby justify the appropriateness of our algorithm. All the co-ordinates are in meters and are measured in reference to the present AP, which is in the centre of the hexagonal cell. We have used memory less Poisson distribution function in determining the speed of the MS at each instant and varied it within a particular range. The initial speed of MS at the origin of call was taken as $20 \mathrm{~m} / \mathrm{s}$, and after the execution the average speed was recorded at $19.35 \mathrm{~m} / \mathrm{s}$ and the speed variation is shown in Figure 9.

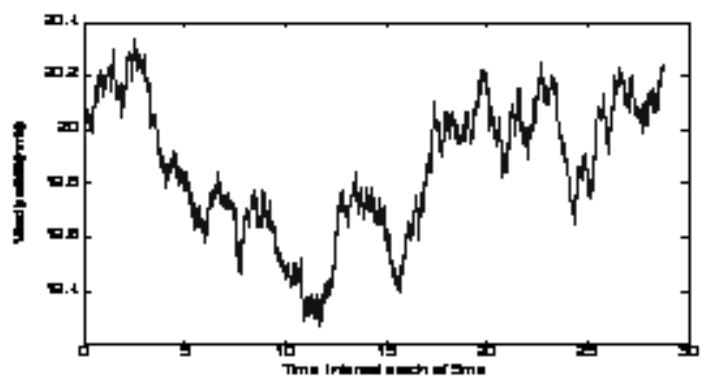

Figure 9. The speed of the MS at each instant of time. As proposed when the RSSI falls below a certain threshold (here we have considered it to be equal to $20 \mathrm{~dB}$ ), the algorithm stops and the scanning starts. But the final handoff procedure is completed only when the hysteresis constant is realised. In Figure 10 we have considered a parabolic trajectory of an MS. Comparing with Figure 7 we can see that 11 positions of the MS are taken into consideration, and corresponding to each point the probable APs are predicted.

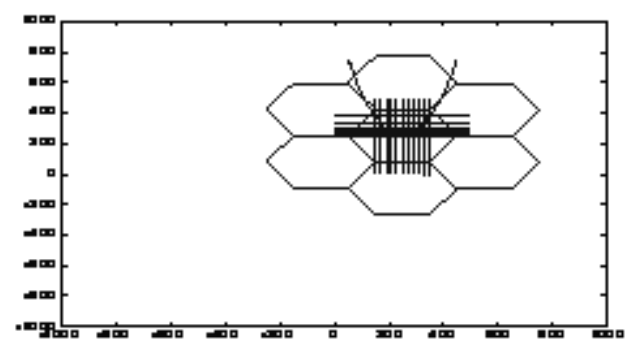

Figure 10. Trajectory of MS

When the conditions are met our algorithm predicts the target AP to be AP2, which is true as can be viewed from Figure 10. Now in Figure 11 we have created a totally random trajectory of MS based on real time consideration. Here a situation arises when the received RSSI goes below the threshold value, but as 
the hysteresis condition is not realized within the maximum scanning time so handoff does not occur to the AP3, and thereby the probability of false handoff is also reduced by our algorithm. And in this case handoff occurs finally with AP2. A set of sample runs were made by varying the parameters such as cell coverage area or initial velocity of MS and each time the AP was predicted correctly with only one AP as the potential AP to which the handoff occurs.

For the co-ordinate evaluation method the coverage region of the AP is taken as regular hexagons of length $231 \mathrm{~m}$ approx (which satisfy the topological conditions of an AP in urban area). At the end of the algorithm we note the range of co-ordinate or the range of angles in which the MS may lie after handover and we compared this with its actual co-ordinate and angle that it makes with the previous AP. The result of this simulation justifies the appropriateness of our algorithm The average scan phase was taken as $50 \mathrm{~ms}$.

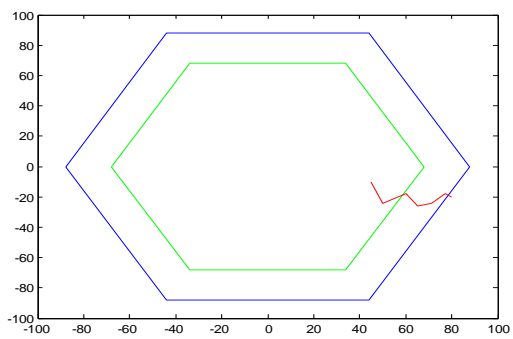

Figure 11. Trajectory of the MS

All the co-ordinates are in meters and are measured in reference to the present AP as the origin and axis shown as in Figure 12. The average speed of the MS was recorded as $19.0222 \mathrm{~m} / \mathrm{s}$ after the origination of call. As proposed, when it reaches the boundary, as shaded in Figure 12 the algorithm stops. In this sample run incorporated with our algorithm, for co-ordinate evaluation method we get the range of expected co-ordinate in which the MS may lie after our algorithm is found to be 199.9070 to 199.9185 for $x$ co-ordinate and 77.4786 to 77.4862 for y co-ordinate (co-ordinates measured according to the system of axis as in Figure 4). The actual $\mathrm{x}$ co-ordinate is found to be 199.9117, and the actual y co-ordinate is determined to be 77.4825 , which lie within our expected range of co-ordinates. This indicates that it is heading towards AP 1 (refer to Figure 4) as the expected range of co-ordinates lie in the coverage area of AP 1. So only the channel associated with AP1 may be scanned during handover, which reduces the handover latency to a great extent. After the handover phase, the recorded actual co-ordin ate of the MS is found to be (199.9117, 77.4825). For angular displacement method we get the range of expected angle of the MS from 21.1849 to 21.1857. This indicates that it is heading towards AP 1 (refer to Figure 5) as the expected range of angles lie between 0 and 60 degrees. So only the channel corresponding to AP 1 may be scanned during handover which reduces the handover latency to a great extent. After the handover phase, the recorded actual angle that the MS makes with the previous AP is found to be 21.1855 .

This signifies the appropriateness of both the algorithm. A set of sample runs were made using this algorithm by varying the parameters like cell coverage area or the speed of the MS which yielded the result that for angular displacement method $99.25 \%$ and for co-ordinate evaluation method $98.36 \%$ of cases, the correct AP or APs were identified. For both the algorithms for less than $5 \%$ of the cases, there were two APs to be scanned and for those cases the scanning time was taken double of the scanning time of each AP. The expected X co-ordinate of the MS and its actual X co-ordinate is plotted in Figure 12 and the same for $\mathrm{Y}$ co-ordinate for any 5 second time interval is plotted in Figure13. For angular displacement method the expected and the actual angle is plotted in the Figure 14 for any 5 second time interval. From all the three mathematical algorithms area comparison method proves to be the most effective algorithm in determining the next potential AP. We made a sample run of our simulation and calculated the time required for handoff at regular intervals of time. We calculated 100 such instances and calculated the total time required for the handoff to take place. From a particular sample run we got the average time delay for all 100 instances as $4.1129 \mathrm{~ms}, 4.98 \mathrm{~ms}$ and $5.04 \mathrm{~ms}$ for area comparison method, co-ordinate evaluation method and an gular displacement method respectively.

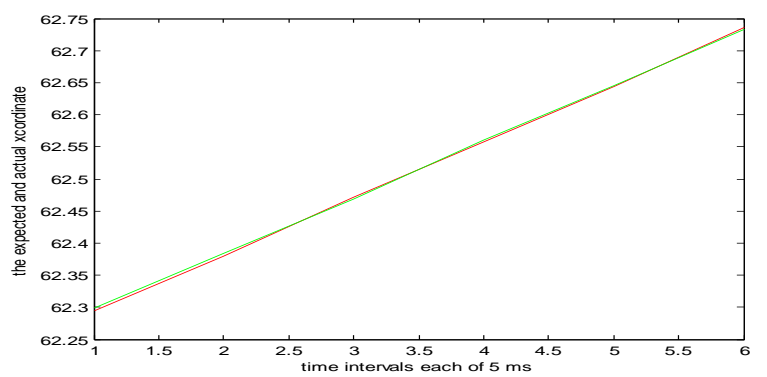

Figure 12.A magnified region of any 5 second time interval in which the expected and the actual x co-ordinate (in meters) are plotted.

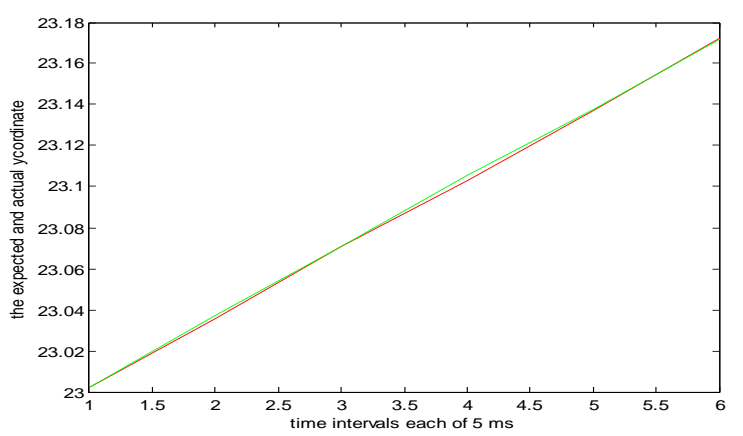

Figure 13.A magnified region of any 5 second time interval in which the expected and the actual y co-ordinate (in meters) are plotted

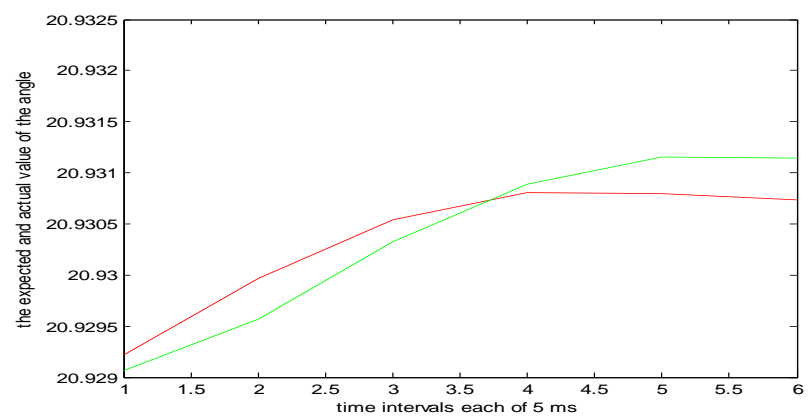

Figure 14.A magnified region of any 5 second time intervals in which the expected and the actual angles (in degrees) are plotted. 
Our results for each mathematical model it is better than the previous results as in [6] and [10] with area comparison method being the most effective algorithm in predicting the next potential AP and hence has a much lower value of handoff delay. Thus the proposed algorithm effectively reduces the handoff algorithm to a great extend.

\section{CONCLUSION}

Our discussion is based on IEEE $802.11 \mathrm{~b}$ standard, even though the proposed set-up is also valid for IEEE $802.11 \mathrm{~g}$ with minor adjustments. As is evident, the discovery phase is still the most time consuming phase of the handoff process. Future simulations may be done using different topologies with modifications regarding selective scanning and preauthentication using IAPP. Improvements in the mathematical models, as proposed, to find the next potential AP with lower computational expense is also very much desirable.

Moreover, our method works best in networks where FDMA is used for multiple accesses. Though networks using TDMA has also reduced handoff latency by this method, but still in the best case scenario, the net time delay is a bit more than that of networks using FDMA. So, further research work can be done in this field. We have considered link layer handoff delay only. For intra-system handoff, a bit more time will be required due to increase in message processing delay. Though we have nullified the authentication delay, yet the re-association delay cannot be neglected while doing the simulations. But for this case, as the re-association delay is very small as compared to the handoff delay, so we can neglect it for our experimental results

\section{REFERENCES}

[1] "Wireless LAN Medium Access Control (MAC) and Physical Layer (PHY) Specifications," IEEE Standards, 1999

[2] Wankawee Puangkor, Panita Pongpaibool, "A Survey of Techniques for Reducing Handover Latency and Packet Loss in Mobile IPv6"

[3] Hye-Soo Kim, Sang Hee Park, Chun-Su Park, Jae Won Kim and Sung-Jea Ko. "Selective Channel Scanning for Fast Handoff in Wireless LAN using Neighbor Graph", July 2004

[4] Jin Teng, Changqing Xu, Weijia Jia, Dong Xuan. "D-scan: Enabling Fast and Smooth Handoffs in AP-dense 802.11 Wireless Networks

[5] S. Kyriazakos, D. Drakoulis and G. Karetsos,'Optimazation of the Handover Algorithm based on the "Position of the Mobile Terminals" in Proceedings of Symposium on Communications and Vehicular Technology, October 2000

[6] J. Pesola and S. Pokanen, "Location-aided Handover in Heterogeneous Wireless Networks," in Proceedings of Mobile Location Workshop, May 2003.

[7] V. Bahety and R. Pendse, "Scalable QoS Provisioning for Mobile Networks using Wireless Sensors," in Proceedings of IEEE International Conference on Wireless Communications and Networking (WCNC'04), March 2004.
[8] Hongqiang Zhai, Xiang Chen, and Yuguang Fang. How well can the IEEE 802.11 wireless lan support quality of service? IEEE Transactions on Wireless Communications, 4(6):3084-3094, December 2005

[9] Ping-Jung Huang, Yu-Chee Tseng. "A Fast Handoff Mechanism for IEEE 802.11 and IAPP Networks"

[10] Minho Shin, Arunesh Mitra and William A. Arbaugh. Improving the latency of 802.11 handoffs using neighbour graphs. In Mobisys '04:proceedings of the 2nd international conference on mobile systems, applications and services,pages 70-83, New York,USA,2004. ACM press.

[11] Chung-Sheng Li et.al. 'A neighbor caching mechanism for handoff in IEEE 802.11 Wireless networks.' Springer 20 March 2008,DOI 10.1007/s11227-008-0175-3

[12] M.Ohta, "Smooth Handover over IEEE 802.11 Wireless LAN,"Internet Draf: draft-ohta- smooth-handover-wlan00.txt, Jun. 2002.

[13] Arunesh Mishra, Minho Shin and William Arbaugh. An empirical analysis of the IEEE 802.11 MAC layer handoff process. SIGCOMM comput.common.rev,33 (2):93102,2003 .

[14] S. Park and Y. Choi. Fast inter-ap handoff using predictiveauthentication scheme in a public wireless lan. Networks2002 (Joint ICN 2002 and ICWLHN 2002), August 2002.

[15] S. Shin, A. Forte, A. Rawat, and H. Schulzrinne, "Reducing MAC Layer Handoff Latency in IEEE 802.11 Wireless LANs," in Proc. ACM MobiWac 2004, October 2004.

[16] Ashutosh Dutta ,S Madhani, Wai Chen, "GPS-IP based fast Handoff for Mobiles"

[17] Chien-Chao Tseng, K-H Chi, M-D Hsieh and H-H Chang," Location-based Fast Handoff for 802.11 Networks",IEEE COMMUNICATIONS LETTERS,VOL9, NO 4 April 2005.

[18] Yogesh Ashok Powar and Varsha Apte, "Improving the IEEE 802.11 MAC Layer Handoff Latency to Support Multimedia Traffic"

[19] Eun-Dae Kim, Duck-Ki Ahn, Su-Yong Kim, and SungJoon Cho"Improvement of Pre-authentication with Neighbor Graph for Fast Handoff in WLANs"

[20] D. Johnson, C. Perkins, and J. Arko, "Mobility Support in IPv6, Internet Engineering Task Force Request for Comments (RFC) 3775," June 2004.

[21] J. Montavont, N. Montavont, and T. Noel, "Enhanced schemes for L2 handover in IEEE 802.11 networks and their evaluations," in Proceedings of the IEEE International Symposium on Personal Indoor and Mobile Radio Communications (PIMRC'05), September 2005.

[22] C. Perkins and K.-Y. Wang, "Optimized smooth handoffs in Mobile IP," in Proceedings of IEEE International Sy mposium on Computers 
[23] N. Montavont and T. Noel, "Anticipated Handover over IEEE 802.11 Networks," in Proceedings of the IEEE International Conference on Wireless and Mobile Computing, Networking and Communications (WiM ob'2005), August 2005.
[24] J. Montavont and T. Noel, “ IEEE 802.11 Handovers Assisted by GPS Information”, IEEE 1-4244-04959/06,2006

[25] M.S.Gast," 802.11 wireless networks, the definitive guide," O'Reilly \& associates,USA,2002,pp.114-137. 